\title{
Chemical Characterization of Metabolites from the Husk of Theobroma cacao by GC-MS in Cuba
}

\author{
Danae Perez \\ Department of Foods, Faculty of Pharmacy and Foods, Havana University, Cuba
}

José González

Department of Pharmacy, Faculty of Pharmacy and Foods, Havana University, Cuba

Enrique Gómez

Department of Pharmacy, Faculty of Pharmacy and Foods, Havana University, Cuba

Julio Perez

National Center of Toxicology, Military Hospital "Dr. Carlos J. Finlay", Havana, Cuba

José L. Rodríguez

Research Institute of Food Industry, Havana, Cuba

Max Monan

ARVARNAM, Martinica, France

Received: April 23, $2018 \quad$ Accepted: June 6, $2018 \quad$ Published: June 8, 2018

Doi: 10.5296/jab.v6i2.13263 URL: http://doi.org/10.5296/jab.v6i2.13263

\begin{abstract}
An ethanolic extract at $90 \%$ made by Soxhlet was analyzed using a Shimadzu spectrometer coupled to gas chromatography (GCMS-QP2010 Ultra) after derivatization with BSTFA from the husk of Theobroma cacao. The drug powdered was evaluated through a phytochemical
\end{abstract}


screening to get information about the chemical components present in the sample. Ethereal, ethanolic and aqueous extract were analyzed by the same GCMS technique. Gas Chromatography Mass Spectrometry (GC-MS) analysis revealed the presence of 74 metabolites from this husk. According to NIST21 and NIST107 Libraries Databases some of the phyto-compounds screened were Caffeine, Theobromine, Catechine, Uridine, Stigmasterol, Cholesterol and Androstane-11,17-dione,3-[(trimethylsilyl)oxy]-,17-[O-(phenyl methyl)oxime]. Phytochemical screening revealed the possible presence of fats, alkaloids, triterpenes and steroids, catechins, reductants sugars, saponins, phenolic compounds, aminoacids or amines, quinones, anthocyanidins, flavonoids and bitter or astringent principles.

Keywords: Theobroma cacao, GC-MS, Phytochemical screening, Metabolites, Derivatization

\section{Introduction}

Cacao, (Theobroma cacao), also called cocoa, tropical evergreen tree (family Malvaceae, formerly Sterculiaceae) grown for its edible seeds, whose scientific name means "food of the gods" in Greek. Native to lowland rainforests of the Amazon and Orinoco river basins, cacao is grown commercially in the New World tropics as well as western Africa and tropical Asia. Its seeds, called cocoa beans, are processed into cocoa powder, cocoa butter, and chocolate (Cook, 2018).

Cacao grows in the forest understory to a height of 6-12 meters (20-40 feet), usually remaining at the lower end of this range. It's oblong leathery leaves measure up to $30 \mathrm{~cm}$ (12 inches) in length, and are periodically shed and replaced by new leaves that are strikingly red when young. Its flowers are either foul-smelling or odorless; they can be present at all times but appear in abundance twice a year. These flowers grow in clusters directly from the trunk and limbs and are about $1 \mathrm{~cm}(0.4 \mathrm{inch})$ in height and breadth. They can be white, rosy, pink, yellow, or bright red, depending on the variety, and are pollinated by tiny flies called midges in many areas (Ronse, 2010).

After four years the mature cacao tree produces fruit in the form of elongated pods; it may yield up to 70 such fruits annually. The pods, or cherelles, range in color from bright yellow to deep purple (Figure 1). They ripen in less than six months to a length up to $35 \mathrm{~cm}$ (14 inches) and a width at the center of $12 \mathrm{~cm}$ (4.7 inches). Each pod has numerous ridges running along its length and holds 20 to 60 seeds, or cocoa beans, arranged around the long axis of the pod. The oval seeds are about $2.5 \mathrm{~cm}$ ( 1 inch) long and are covered with a sweet sticky white pulp (Clement et al., 2010). 


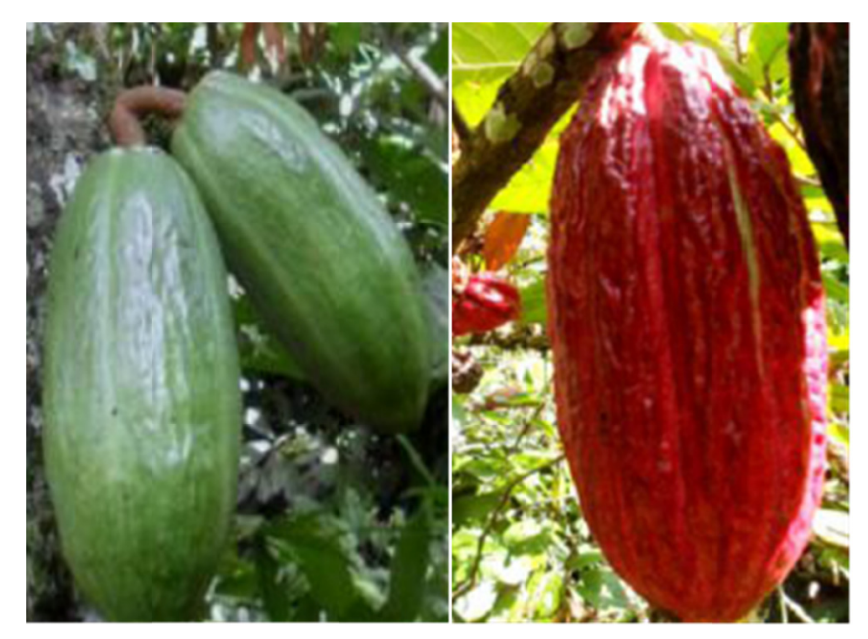

Figure 1. Cacao's elongated pods

Cacao thrives at altitudes of 30 to 300 meters (100 to 1,000 feet) above sea level in areas where temperatures do not range much below $20^{\circ} \mathrm{C}\left(68^{\circ} \mathrm{F}\right)$ or above $28^{\circ} \mathrm{C}\left(82{ }^{\circ} \mathrm{F}\right)$. Rainfall requirements depend upon the frequency and distribution of rain and the degree of water retention by the soil; the minimum necessary rainfall is about $100 \mathrm{~cm}$ (39 inches) evenly distributed throughout the year, but 150-200 cm (59-79 inches) is optimal. Successful cultivation also requires deep well-drained soil that is porous and rich in humus. Protection against strong winds is necessary because of the tree's shallow root system (FAO, 2017).

In the cocoa bean industry, some by-products go underutilized. Some of these components could provide other innovative products, and such is the case with the husk of the cocoa bean. Previous studies have attributed the husk with a high antioxidant capacity, which added to its relative low cost, makes it an attractive ingredient for the production of infusions. However, prior to promoting it as such, its quality needs to be guaranteed (Sangronis et al., 2014). The aim of this study was to evaluate the chemical composition of the husk of cocoa bean that grows in Cuba which is much utilized in the Cuban chocolate industry.

\section{Material and Methods}

\subsection{Plant Material}

The sample was the husk of cocoa bean after its separation from the fruits. It was supplied in 2018 by the Chocolate Factory located in Baracoa, Guantanamo Province, Cuba. After the collection the husks were packet in nylon bags without elimination of foreign matters. The material was grounded in a high-speed hammer-mill. The sample keeps its brown color (Fig. 2) and a very nice chocolate's smell. 


\section{Macrothink}

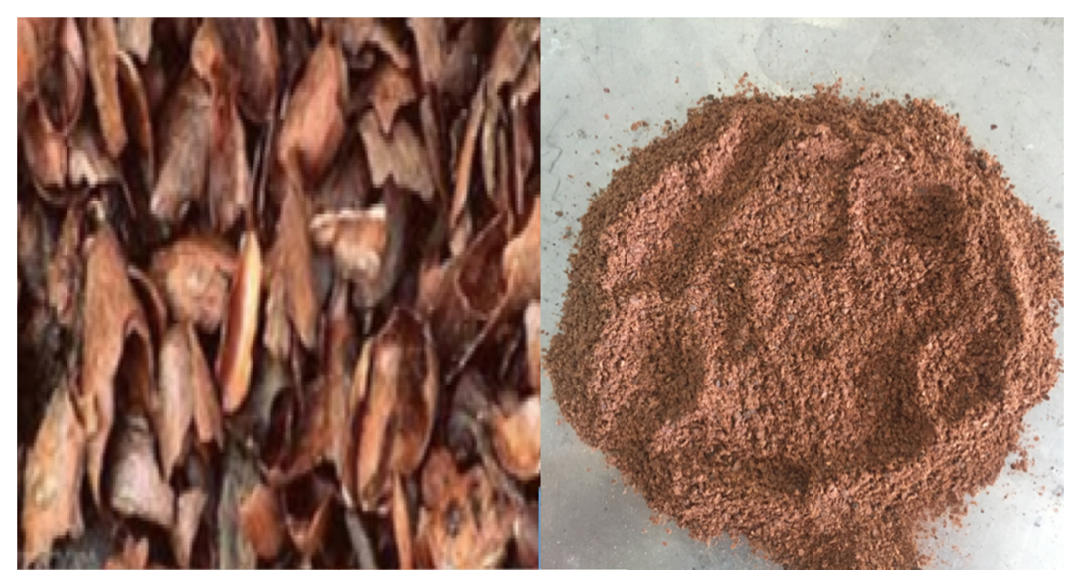

Figure 2. Husk of cocoa beans (after separation and powdered)

\subsection{Phytochemical Screening}

The bioactive compounds were screened to ascertain their presences in diethyl ether, ethanol and water according to Chhabra et al., 1989.

\subsection{Extract Preparation}

The extracts were prepared with the ground material $(60 \mathrm{~g})$ without screen extracted in a Soxhlet apparatus with $675 \mathrm{~mL}$ of ethanol at $90 \%$ during 20 hours. The ethanolic extracts were concentrated and evaporated under vacuum to $200 \mathrm{~mL}$ at $120 \mathrm{rpm}$, a temperature of $70{ }^{\circ} \mathrm{C}$ and 500 mbar.

\subsection{Procedures, Instrumentation and Parameters}

The sample were subjected to chromatographic analysis in equipment GC/MS, brand Shimadzu QP2010, equipped with a splitter split/splitless. With a BP5 (30 m $\times 0.25 \mathrm{~mm} \times$ 0.25 microns) capillary column under the following chromatographic conditions: Helium gas carrier obtained by electron impact fragments to a power of $70 \mathrm{eV}$ rate of $1.2 \mathrm{~mL} / \mathrm{min}, 1: 50$ split flow and the volume of injected sample of $1 \mathrm{ul}$. Programmed oven temperature: initial temperature was $70{ }^{\circ} \mathrm{C}$ with a heating ramp of $10{ }^{\circ} \mathrm{C} / \mathrm{min}$ to $300{ }^{\circ} \mathrm{C}$ and remained stable at this temperature for 10 minutes. Subsequently the temperature was increased at a rate of $10{ }^{\circ} \mathrm{C} /$ minute to $300{ }^{\circ} \mathrm{C}$ for a total time of 78 minutes with an injector temperature $250{ }^{\circ} \mathrm{C}$ and the interface temperature $300^{\circ} \mathrm{C}$. The compounds were analyzed using GC/MS NIST2 1 and NIST107 library and having into account the results obtained after phytochemical screening according with Gómez (2017). Silylation agent was N, O-bis (trimethylsilyl) trifluoroacetamide (BSTFA) CAS 25561-30-2 Lot: 0901-1 Macherey-Nagel GmbH \& C. KG. Chromatographic running was done four times: ethanolic extract by Soxhlet at $90 \%$ and with the ethereal, ethanolic and aqueous extracts by separated getting in the phytochemical screening.

\section{Results and Discussion}

\subsection{Phytochemical Screening}

Preliminary phytochemical screening showed the presence of fats and oils, alkaloids, tannins and/or phenolic compounds, flavonoids, catechins, triterpenes and/or steroids, reductants sugars, aminoacids and/or amines, quinones, anthocyanin, saponins and bitter principles. The 
test for coumarins and lactones was not $100 \%$ confident, although showed some turbidity (Table 1).

Table 1. Phytochemical screening of the cacao husk

\begin{tabular}{|c|c|c|c|}
\hline Test for constituent groups & Ether & Ethanol & Water \\
\hline Sudan & + & & \\
\hline Dragendorff & - & ++ & + \\
\hline Wagner & - & ++ & + \\
\hline Baljet & - & \pm (turbidity) & \\
\hline Liebermann-Burchard & + & + & \\
\hline Catechins & & + & \\
\hline Resins & & - & \\
\hline Fehling & & + & + \\
\hline Foam & & + & + \\
\hline $\mathrm{FeCl}_{3}$ & & $+($ dark green $)$ & $+($ dark green $)$ \\
\hline Ninhydrin & & + & \\
\hline Börntrager & & $+++($ red $)$ & \\
\hline Kedde & & - & \\
\hline Anthocyanin & & + & \\
\hline Shinoda & & + & + \\
\hline Bitter and astringent principles & & & bitter \\
\hline
\end{tabular}

\subsection{GC/MS Analyses}

From ethanolic extract at 90\%, seventy-four different chemical components were characterized from the husk of Theobroma cacao L. by GC/MS experiments in Cuba for the first time. Among them 11 aminoacids, 35 different kind of acids, 13 different kind of sugars or related compounds, one aldehyde, 3 sterols or related compounds, two alkaloids, one catechine, one purine, one pyrimidine, one lactone, one alkane, 3,4-dihydroxyphenylethylamine, 2,6-deoxyfructosazine, 1-monooleoylglycerol trimethylsilyl ether and 1,3-dipalmitin trimethylsilyl ether (Table 2). According with Rohloff in 2015, all those kinds of metabolites can be found and characterized using derivatization techniques in combination with GC-MS-Based Metabolite Profiling.

Chromatographic profile of cacao husk showed the same amount of chemical components with few differences up to 46 minutes of retention times in the Soxhlet extraction, ethereal and hydrolacoholic extracts (Figure 3). According with chromatographic profiles aqueous extraction gets the fewer amounts of chemical compounds, indicating that the components have a low or medium polarity. 


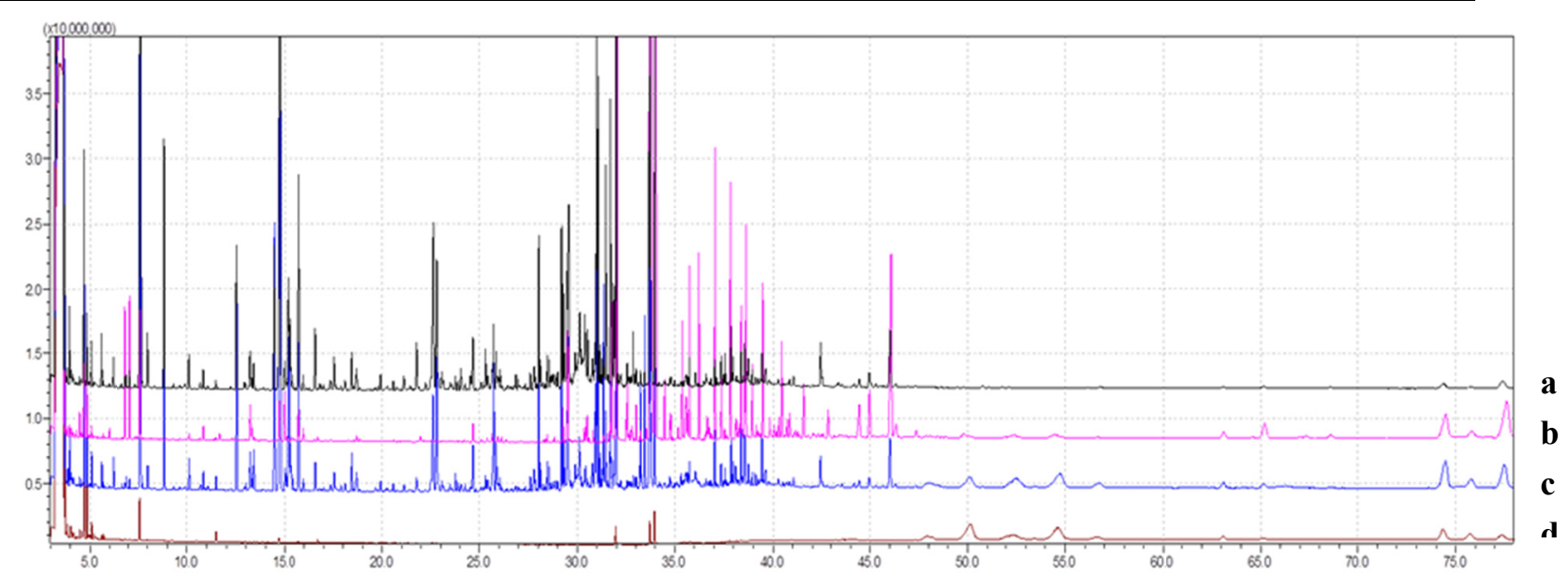

Figure 3. Chromatographic profiles of husk from Theobroma cacao in Cuba (a-Soxhlet; b-hydrolacoholic; c-ether, d-water).

Qualitative analysis indicated that caffeine is the majoritarian alkaloid. Theobromine was found in lower concentrations. Both results are according to Cuéllar et al., 2012, although in this case, theophylline was not found into ethanolic extract at $90 \%$. Figure 4 show the magnified zone in which caffeine and theobromine appears. Table 2 lists the names, retention times, molecular masses, base peaks and chemical formulae of the identified compounds.

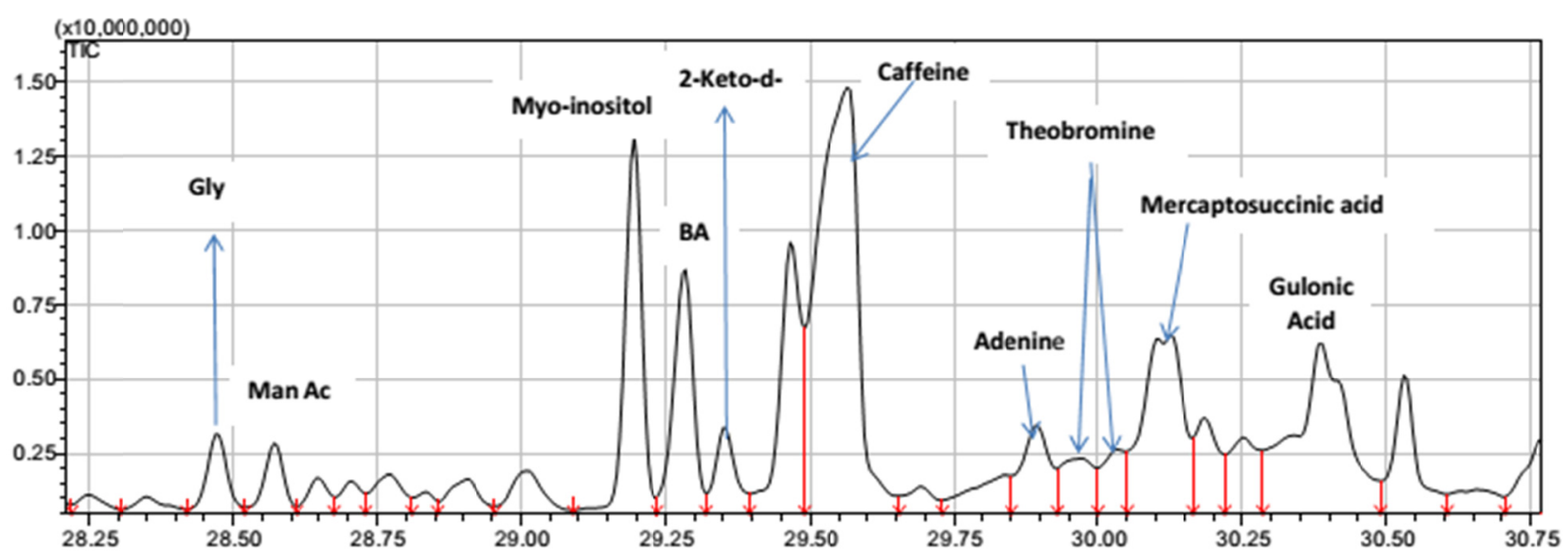

Figure 4. Magnified zone in which appear caffeine and theobromine

(Gly: Glycine; Man Ac: Mannoonic acid; BA: 3, 4-hydroxy-Benzoic acid; 2-Keto-d-gluconic acid).

The results show the great variety of chemical components in this part of the plant that is in general, underutilized in all countries that produce cacao beans in the world, and as can see, may be can used to improve the health in human been or in the food indlustry to make new types of liquors or beverages or in the agricultural to supply animal food or as fertilizer (Sangronis et al., 2014; Cuéllar et al., 2012; Collazos, 2012). The beneficial activities of those compounds are known, in this sense, it is necessary to mention the important role of two of 
those compounds identified in the sample like Dodecanoic and Hexadecanoic acid. Dodecanoic acid is useful as Antioxidant, Antibacterial, COX-1\&COX-2 inhibitor, Antiviral, Hypocholesterolemic, and candidacies, while Hexadecanoic acid can act as Antioxidant, Flavor, Hypocholesterolemic, Nematicide, Pesticide, Lubricant, Antiandrogenic, Hemolytic and 5-Alpha reductase inhibitor (Rajkumar et al., 2016).

Table 2. Chemical compounds identified in Theobroma cacao husk by GC-MS

\begin{tabular}{|c|c|c|c|c|c|}
\hline $\begin{array}{l}\text { Peak } \\
\text { No. }\end{array}$ & $\begin{array}{l}\text { Retention } \\
\text { time }\end{array}$ & $\begin{array}{l}\text { Molecular } \\
\text { Mass }\end{array}$ & $\begin{array}{l}\text { Base } \\
\text { peak }\end{array}$ & Compound Name & $\begin{array}{l}\text { Molecular } \\
\text { formula }\end{array}$ \\
\hline 1 & 7.643 & 234 & 73 & Propanoic acid & $\mathrm{C}_{9} \mathrm{H}_{22} \mathrm{O}_{3} \mathrm{Si}_{2}$ \\
\hline 2 & 7.935 & 220 & 73 & Acetic acid & $\mathrm{C}_{8} \mathrm{H}_{20} \mathrm{O}_{3} \mathrm{Si}_{2}$ \\
\hline 3 & 8.843 & 233 & 116 & L-Alanine & $\mathrm{C}_{9} \mathrm{H}_{23} \mathrm{NO}_{2} \mathrm{Si}$ \\
\hline 4 & 10.842 & 262 & 73 & Butanoic acid & $\mathrm{C}_{11} \mathrm{H}_{26} \mathrm{O}_{3} \mathrm{Si}$ \\
\hline 5 & 10.522 & 261 & 144 & L-Valina & $\mathrm{C}_{11} \mathrm{H}_{27} \mathrm{NO}_{2}$ \\
\hline 6 & 13.224 & 276 & 73 & Pentanoic acid & $\mathrm{C}_{12} \mathrm{H}_{28} \mathrm{O}_{3} \mathrm{Si}$ \\
\hline 7 & 14.441 & 275 & 158 & L-Leucine & $\mathrm{C}_{12} \mathrm{H}_{29} \mathrm{NO}_{2}$ \\
\hline 8 & 14.740 & 308 & 73 & Glycerol & $\mathrm{C}_{12} \mathrm{H}_{32} \mathrm{O}_{3} \mathrm{Si}$ \\
\hline 9 & 14.982 & 208 & 73 & Benzenacetic acid & $\mathrm{C}_{11} \mathrm{H}_{16} \mathrm{O}_{2} \mathrm{Si}$ \\
\hline 10 & 15.177 & 275 & 73 & L-Isoleucine & $\mathrm{C}_{12} \mathrm{H}_{29} \mathrm{NO}_{2}$ \\
\hline 11 & 15.263 & 247 & 102 & gamma-Amino butyric acid & $\mathrm{C}_{10} \mathrm{H}_{25} \mathrm{NO}_{2}$ \\
\hline 12 & 15.734 & 262 & 147 & Butanedioic acid & $\mathrm{C}_{10} \mathrm{H}_{22} \mathrm{O}_{4} \mathrm{Si}$ \\
\hline 13 & 17.542 & 321 & 73 & L-Serine & $\mathrm{C}_{12} \mathrm{H}_{31} \mathrm{NO}_{3}$ \\
\hline 14 & 18.459 & 335 & 73 & L-threonine & $\mathrm{C}_{13} \mathrm{H}_{33} \mathrm{NO}_{3}$ \\
\hline 15 & 18.690 & 276 & 73 & Pentanedioic acid & $\mathrm{C}_{11} \mathrm{H}_{24} \mathrm{O}_{4} \mathrm{Si}$ \\
\hline 16 & 19.934 & 336 & 73 & 3,4-Dihydroxybutanoic acid & $\mathrm{C}_{13} \mathrm{H}_{32} \mathrm{O}_{4} \mathrm{Si}$ \\
\hline 17 & 21.776 & 350 & 73 & Malic acid & $\mathrm{C}_{13} \mathrm{H}_{30} \mathrm{O}_{5} \mathrm{Si}$ \\
\hline 18 & 22.624 & 273 & 156 & L-Proline & $\mathrm{C}_{11} \mathrm{H}_{23} \mathrm{NO}_{3}$ \\
\hline 19 & 22.804 & 336 & 73 & Butanal & $\mathrm{C}_{13} \mathrm{H}_{32} \mathrm{O}_{4} \mathrm{Si}$ \\
\hline 20 & 24.038 & 424 & 73 & 2,3,4-Trihydroxybutiric acid & $\mathrm{C}_{16} \mathrm{H}_{40} \mathrm{O}_{5} \mathrm{Si}$ \\
\hline 21 & 24.689 & 310 & 73 & Benzenepropanoic acid & $\mathrm{C}_{15} \mathrm{H}_{26} \mathrm{O}_{3} \mathrm{Si}$ \\
\hline 22 & 25.665 & 282 & 73 & Benzoic acid & $\mathrm{C}_{13} \mathrm{H}_{22} \mathrm{O}_{3} \mathrm{Si}$ \\
\hline 23 & 25.700 & 438 & 73 & D-Arabinose & $\mathrm{C}_{17} \mathrm{H}_{42} \mathrm{O}_{5} \mathrm{Si}$ \\
\hline 24 & 25.742 & 309 & 218 & L-phenylalanine & $\mathrm{C}_{15} \mathrm{H}_{27} \mathrm{NO}_{2}$ \\
\hline 25 & 25.888 & 363 & 102 & 3,4-Dihydroxyphenylethylamine & $\mathrm{C}_{17} \mathrm{H}_{35} \mathrm{NO}_{2}$ \\
\hline
\end{tabular}




\begin{tabular}{|c|c|c|c|c|c|}
\hline 26 & 26.085 & 364 & 73 & Arabinoic acid & $\mathrm{C}_{14} \mathrm{H}_{32} \mathrm{O}_{5} \mathrm{Si}$ \\
\hline 27 & 26.873 & 318 & 73 & Isocitric lactone & $\mathrm{C}_{12} \mathrm{H}_{22} \mathrm{O}_{6} \mathrm{Si}$ \\
\hline 28 & 27.627 & 512 & 73 & Ribitol & $\mathrm{C}_{20} \mathrm{H}_{52} \mathrm{O}_{5} \mathrm{Si}$ \\
\hline 29 & 27.790 & 524 & 73 & Myoinositol & $\mathrm{C}_{21} \mathrm{H}_{52} \mathrm{O}_{5} \mathrm{Si}$ \\
\hline 30 & 27.47 & 512 & 73 & Xylitol & $\mathrm{C}_{20} \mathrm{H}_{52} \mathrm{O}_{5} \mathrm{Si}$ \\
\hline 31 & 28.467 & 287 & 73 & Glycine & $\mathrm{C}_{12} \mathrm{H}_{25} \mathrm{NO}_{3}$ \\
\hline 32 & 28.587 & 452 & 73 & 2-Deoxy-galactose & $\mathrm{C}_{18} \mathrm{H}_{44} \mathrm{O}_{5} \mathrm{Si}$ \\
\hline 33 & 28.709 & 333 & 75 & L-Glutamic acid & $\mathrm{C}_{13} \mathrm{H}_{27} \mathrm{NO}_{5}$ \\
\hline 34 & 28.771 & 526 & 73 & Ribonic acid & $\mathrm{C}_{20} \mathrm{H}_{50} \mathrm{O}_{6} \mathrm{Si}$ \\
\hline 35 & 28.906 & 364 & 73 & D-Arabinonic acid & $\mathrm{C}_{14} \mathrm{H}_{32} \mathrm{O}_{5} \mathrm{Si}$ \\
\hline 36 & 29.289 & 370 & 73 & 3,4-hydroxy-Benzoic acid & $\mathrm{C}_{16} \mathrm{H}_{30} \mathrm{O}_{4} \mathrm{Si}$ \\
\hline 37 & 29.355 & 554 & 73 & 2-Keto-d-gluconic acid & $\mathrm{C}_{21} \mathrm{H}_{50} \mathrm{O}_{7} \mathrm{Si}$ \\
\hline 38 & 29.497 & 466 & 73 & Mannoonic acid & $\mathrm{C}_{18} \mathrm{H}_{42} \mathrm{O}_{6} \mathrm{Si}_{4}$ \\
\hline 39 & 29.5663 & 194 & 194 & Caffeine & $\mathrm{C}_{8} \mathrm{H}_{10} \mathrm{~N}_{4} \mathrm{O}_{2}$ \\
\hline 40 & 29.899 & 279 & 264 & 9H-Purine-6-amine & $\mathrm{C}_{11} \mathrm{H}_{21} \mathrm{~N}_{5} \mathrm{Si}$ \\
\hline 41 & 30.029 & 180 & 180 & Theobromine & $\mathrm{C}_{7} \mathrm{H}_{8} \mathrm{~N}_{4} \mathrm{O}_{2}$ \\
\hline 42 & 30.118 & 366 & 73 & Mercaptosuccinic acid & $\mathrm{C}_{13} \mathrm{H}_{30} \mathrm{O}_{4} \mathrm{Si}$ \\
\hline 43 & 30.388 & 466 & 73 & Gulonic acid & $\mathrm{C}_{13} \mathrm{H}_{42} \mathrm{O}_{6} \mathrm{Si}$ \\
\hline 44 & 30.910 & 397 & 218 & L-Tyrosine & $\mathrm{C}_{18} \mathrm{H}_{35} \mathrm{NO}_{3}$ \\
\hline 45 & 30.986 & 410 & 73 & 1,2,3,4-tetrahydroxy-Butane & $\mathrm{C}_{16} \mathrm{H}_{42} \mathrm{O}_{4} \mathrm{Si}$ \\
\hline 46 & 31.063 & 614 & 73 & D-Mannitol & $\mathrm{C}_{24} \mathrm{H}_{62} \mathrm{O}_{6} \mathrm{Si}$ \\
\hline 47 & 31.158 & 614 & 73 & d-Glucitol & $\mathrm{C}_{24} \mathrm{H}_{62} \mathrm{O}_{6} \mathrm{Si}$ \\
\hline 48 & 31.340 & 284 & 88 & Palmitic acid & $\mathrm{C}_{18} \mathrm{H}_{36} \mathrm{O}_{2}$ \\
\hline 49 & 31.453 & 540 & 217 & beta-D-Galactofuranose & $\mathrm{C}_{21} \mathrm{H}_{52} \mathrm{O}_{6} \mathrm{Si}_{5}$ \\
\hline 50 & 31.585 & 540 & 73 & D-Glucose & $\mathrm{C}_{21} \mathrm{H}_{52} \mathrm{O}_{6} \mathrm{Si}_{5}$ \\
\hline 51 & 31.735 & 326 & 73 & Palmitelaidic acid & $\mathrm{C}_{19} \mathrm{H}_{38} \mathrm{O}_{2} \mathrm{Si}$ \\
\hline 52 & 31.833 & 438 & 217 & D-Ribofuranose & $\mathrm{C} 17 \mathrm{H} 42 \mathrm{O} 5 \mathrm{Si}$ \\
\hline 53 & 31.946 & 628 & 73 & Galactonic acid & $\mathrm{C}_{24} \mathrm{H}_{60} \mathrm{O}_{7} \mathrm{Si}_{6}$ \\
\hline 54 & 31.975 & 328 & 73 & Hexadecanoic acid & $\mathrm{C}_{19} \mathrm{H}_{40} \mathrm{O}_{2} \mathrm{Si}$ \\
\hline 55 & 32.183 & 612 & 73 & Inositol & $\mathrm{C}_{24} \mathrm{H}_{60} \mathrm{O}_{6} \mathrm{Si}_{6}$ \\
\hline 56 & 33.005 & 272 & 73 & Dodecanoic acid & $\mathrm{C}_{15} \mathrm{H}_{32} \mathrm{O}_{2} \mathrm{Si}$ \\
\hline 57 & 32.235 & 184 & 55 & 10-Undecenoic acid & $\mathrm{C}_{11} \mathrm{H}_{20} \mathrm{O}_{2}$ \\
\hline 58 & 33.479 & 326 & 88 & Nonadecanoic acid, ethyl ester & $\mathrm{C}_{21} \mathrm{H}_{42} \mathrm{O}_{2}$ \\
\hline
\end{tabular}




\section{Macrothink}

Journal of Applied Biotechnology

ISSN 2327-0640

2018, Vol. 6, No. 2

\begin{tabular}{|c|c|c|c|c|c|}
\hline 59 & 33.702 & 352 & 75 & 9,12-Octadecadienoic acid & $\mathrm{C}_{21} \mathrm{H}_{40} \mathrm{O}_{2} \mathrm{Si}$ \\
\hline 60 & 33.757 & 354 & 117 & Oleic acid & $\mathrm{C}_{21} \mathrm{H}_{42} \mathrm{O}_{2} \mathrm{Si}$ \\
\hline 61 & 33.987 & 356 & 73 & Octadecanoic acid & $\mathrm{C}_{21} \mathrm{H}_{44} \mathrm{O}_{2} \mathrm{Si}$ \\
\hline 62 & 35.753 & 384 & 73 & Eicosanoic acid & $\mathrm{C}_{23} \mathrm{H}_{48} \mathrm{O}_{2} \mathrm{Si}$ \\
\hline 63 & 36.056 & 460 & 73 & Uridine & $\mathrm{C}_{18} \mathrm{H}_{36} \mathrm{~N}_{2} \mathrm{O}_{6} \mathrm{Si}_{3}$ \\
\hline 64 & 37.578 & 846 & 73 & D-Turanose & $\mathrm{C}_{33} \mathrm{H}_{78} \mathrm{O}_{11} \mathrm{Si}_{7}$ \\
\hline 65 & 37.877 & 808 & 73 & 2,6-Deoxyfructosazine & $\mathrm{C}_{33} \mathrm{H}_{76} \mathrm{~N}_{2} \mathrm{O}_{7} \mathrm{Si}_{7}$ \\
\hline 66 & 38.385 & 500 & 73 & 1-Monooleoylglycerol trimethylsilyl ether & $\mathrm{C}_{27} \mathrm{H}_{56} \mathrm{O}_{4} \mathrm{Si}_{2}$ \\
\hline 67 & 38.552 & 992 & 73 & Per-O-trimethylsilyl-(3-O-.beta.-d-mannopyranosyl-d-glucitol) & $\mathrm{C}_{39} \mathrm{H}_{96} \mathrm{O}_{11} \mathrm{Si}_{9}$ \\
\hline 68 & 38.953 & 440 & 73 & Tetracosanoic acid & $\mathrm{C}_{27} \mathrm{H}_{56} \mathrm{O}_{2} \mathrm{Si}$ \\
\hline 69 & 39.448 & 650 & 73 & Catechine & $\mathrm{C}_{30} \mathrm{H}_{54} \mathrm{O}_{6} \mathrm{Si}_{5}$ \\
\hline 70 & 44.975 & 484 & 83 & Stigmasterol & $\mathrm{C}_{32} \mathrm{H}_{56} \mathrm{OSi}$ \\
\hline 71 & 46.030 & 458 & 129 & Cholesterol & $\mathrm{C}_{30} \mathrm{H}_{54} \mathrm{OSi}$ \\
\hline 72 & 46.335 & 481 & 91 & $\begin{array}{l}\text { Androstane-11,17-dione, } \\
\text { methyl)oxime] }\end{array}$ & $\mathrm{C}_{29} \mathrm{H}_{43} \mathrm{NO}_{3} \mathrm{Si}$ \\
\hline 73 & 77.439 & 496 & 75 & $9,12,15$-Octadecatrienoic acid & $\mathrm{C}_{27} \mathrm{H}_{52} \mathrm{O}_{4} \mathrm{Si}_{2}$ \\
\hline 74 & 77.518 & 640 & 371 & 1,3-Dipalmitin trimethylsilyl ether & $\mathrm{C}_{38} \mathrm{H}_{76} \mathrm{O}_{5} \mathrm{Si}$ \\
\hline
\end{tabular}

\section{Conclusions}

The GC-MS study reveals that Theobroma cacao husk contains a number of phytoconstituents which are to be used for various therapeutic purposes or in food industry. In this study, 74 compounds were identified from GCMS data. The compounds identified can be also used as biomarkers especially for $T$. cacao husk because little research has been published for this part of the plant. Chromatographic profiles reveal high predominance of fatty acids, aminoacids and reductants sugars, and two important alkaloids: caffeine and theobromine, which may be considered a source of important phytochemicals with bioactive properties to be explored for pharmaceutical applications. This is the first report with complete GC/MS data for these compounds found in the underutilized husk of Theobroma cacao that grows in Cuba.

\section{ConflicT OF Interest StatemENT}

We declare that we have no conflict of interest.

\section{References}

Cook, L. R. (2018). Cacao tree. Encyclopedia Britannica.

Chhabra, Sc., Uiso, F. C., \& Mshin, E. N. (1989). Phytochemical Screening of Tanzanian Medical Plants. Journal of Ethnopharmacology, 11, 157-179. https://doi.org/10.1016/0378-8741(84)90037-0

Clement, C. R., de Cristo-Araújo, Michelly, d'Eeckenbrugge, Geo Coppens, Alves Pereira, 
Alessandro, Picanço-Rodrigues, Doriane (6 January 2010). Origin and Domestication of Native Amazonian Crops. Diversity, 2(1), 72-106. https://doi.org/10.3390/d2010072

Collazos, Alarcón M. A. (2012). CACAO GRAIN SHELL (Theobroma cacao L.) IN GROWTH RATIONS, FINISHED FOR PIGS.

Cuéllar, G. O., \& Guerrero, A. G. (2012). Antibacterial activity of the cacao bean husk, Theobroma cacao L. Rev. MVZ Córdoba, 17(3), 3176-3183. https://doi.org/10.21897/rmvz.218

FAO. (2017). Cocoa beans production in 2016, Crops/World regions/Cocoa beans/Production quantity from pick lists. United Nations Food and Agriculture Organization, Statistics Division (FAOSTAT). 2017.

José, G. (2017) Pharmacognostic, Physicochemical Standardization and Phytochemical Analysis of Pistils with Pollen from the Flowers of Talipariti Elatum Sw. SF J Herb Med, 1(2).

Rajkumar, P., Selvaraj, S., Suganya, R., Velmurugan, D., \& Kumaresan, S. (2016). GC-MS Characterization of the Anti-Diabetic Compounds From the Flowers of Cassia Auriculata (AVARAM): A structure Based Molecular Docking Studies. International Journal of Innovative Research in Science, Engineering and Technology, 5(1), 85-93.

Rohloff, J. (2015). Analysis of Phenolic and Cyclic Compounds in Plants Using Derivatization Techniques in Combination with GC-MS-Based Metabolite Profiling. Molecules, 20, 3431-3462. https://doi.org/10.3390/molecules20023431

Ronse De Craene, \& Louis, P. (4 February 2010). Floral Diagrams: An Aid to Understanding Flower Morphology and Evolution. Cambridge: Cambridge University Press.

Sangronis, E., Soto, M. J., Valero, Y., \& Buscema, I. (2014). Cascarilla de cacao venezolano como materia prima de infusiones. ARCHIVOS LATINOAMERICANOS DE NUTRICIÓN. Órgano Oficial de la Sociedad Latinoamericana de Nutrición.

\section{Copyright Disclaimer}

Copyright reserved by the author(s).

This article is an open-access article distributed under the terms and conditions of the Creative Commons Attribution license (http://creativecommons.org/licenses/by/3.0/). 